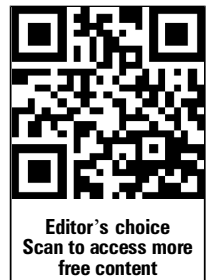

free content

\title{
Current situation for medication process and pharmacovigilance in The Netherlands: a comparison between cytostatic and non-cytostatic agents
}

\author{
B P C van Oijen, ${ }^{1}$ C Mestres Gonzalvo, ${ }^{1}$ R Janknegt, ${ }^{1}$ F P J Peters, ${ }^{2}$ B Winkens, ${ }^{3}$ \\ H C Schouten, ${ }^{4}$ P H M van der Kuy ${ }^{1}$
}

${ }^{1}$ Department of Clinical Pharmacy and Toxicology, Orbis Medical Centre, Sittard, The Netherlands

${ }^{2}$ Department of Haematology and Oncology, Orbis Medical

Centre, Sittard,

The Netherlands

${ }^{3}$ Department of Methodology and Statistics, School for

Public Health and Primary

Care, Maastricht University,

Maastricht, The Netherlands

${ }^{4}$ Department of Internal

Medicine, Division of

Haematology, University

Hospital Maastricht

Maastricht, The Netherlands

\section{Correspondence to}

B P C van Oijen,

Department of Clinical

Pharmacy and Toxicology,

Orbis Medical Centre,

Dr. H van der Hoffplein 1,

Sittard 6162 BG

The Netherlands;

b.vanoijen@orbisconcern.nl

Received 25 February 2013

Revised 29 May 2013

Accepted 30 May 2013

Published Online First

4 July 2013
To cite: van Oijen BPC, Mestres Gonzalvo C, Janknegt $R$, et al. Eur J Hosp Pharm 2013:20: 351-355.

\begin{abstract}
Aim To describe the current situation regarding the medication order entry system and pharmacovigilance for cytostatic and non-cytostatic agents in The Netherlands. Method An electronic questionnaire, consisting of multiple choice questions, was sent to hospital pharmacists to compare general and specific information about cytostatic agents and non-cytostatic agents. A quality index (QI) was established as the gold standard to measure the quality of the medication process phases in the participating hospitals.

Results For cytostatic agents, $17 \%$ of the hospitals used an electronic medication order entry system and in $71 \%$ of these hospitals physicians entered the medication. For non-cytostatic agents, 38\% of the hospitals used an electronic medication order entry system and in 52\% physicians entered the medication. For cytostatic agents, pharmacovigilance for interactions was carried out automatically in 12\% of the hospitals compared with $62 \%$ for non-cytostatic agents.

Pharmacovigilance for cytostatic agents was performed by physicians in $26 \%$ of cases compared with $2 \%$ for non-cytostatic agents. When comparing the QI for participating hospitals, the mean score was $47.2 \%$ ( $95 \% \mathrm{Cl} 44.6 \%$ to $49.9 \%)$. In general, 22 hospitals (39.3\%) scored $\geq 50 \%$.

Concluding statement Pharmacovigilance for interactions and contraindications is not routinely performed for cytostatic agents, while for non-cytostatic agents this is a routine procedure. Keeping in mind the high toxicity and narrow therapeutic range of cytostatic agents, there is still much work to be done to achieve the same pharmacovigilance level and quality that currently exists for non-cytostatic agents.
\end{abstract}

\section{INTRODUCTION}

Drug interactions in oncology are of particular importance owing to the narrow therapeutic index and the inherent toxicity of anticancer agents. Interactions with other medication can cause small changes in the pharmacokinetics or pharmacodynamics of chemotherapy, significantly changing its efficacy or toxicity. ${ }^{1-3}$

The possibility of unintended consequences when using any medication is a well known problem. Commonly these consequences are referred to as adverse drug reactions (ADRs). As a result of clinical trials and post-marketing surveillance, the main and intended effect of a drug is well defined, whereas the effects of ADRs can be either predictable or unpredictable and occur in the short term or long term. ${ }^{4}$

Pharmacovigilance was born in the 20th century as a result of the need to understand as much as possible about any characteristics of ADRs. It is defined as the pharmacological science and activities relating to the detection, assessment, understanding and prevention of adverse effects or any other possible drug-related problems, with a view to the identification of new information about hazards associated with medicines and harm prevention to patients. ${ }^{5}$ Recently, its aims have been widened to include herbs, traditional and complementary medicines, blood products, biologicals, medical devices and vaccines. ${ }^{6}$

ADR reporting is especially important when new agents with limited clinical experience, such as newly released cytostatic agents, enter the marketplace. Initial reports of adverse reactions have taken up to 7 years for trends to begin appearing in the literature. ${ }^{2} 8$ Thus, efforts in post-marketing surveillance have helped in the ability to recognise trends earlier.

Spontaneous reporting is the core data-generating system of international pharmacovigilance, relying on healthcare professionals (and in some cases consumers) to identify and report, almost always voluntarily, any suspected ADR to their national pharmacovigilance centre or to the manufacturer. ${ }^{9}$ However, the reports are anecdotic and the pharmaceutical industry, academics and regulatory agencies need to expand their efforts in monitoring ADRs. ${ }^{10}$ For example, when referring specifically to cytostatic agents, the number of ADRs which may occur is increased due to the large amount of drugs a patients with cancer could be taking for the cancer treatment itself, supportive care drugs and medication for comorbid illnesses. ${ }^{12}$

Optimal pharmacovigilance for intravenous cytostatic agents is important but the efficacy is not clear. $^{711-13}$ Consequently, this questionnaire aims to describe and compare the current situation in The Netherlands for cytostatic and non-cytostatic agents.

\section{METHODS}

A quality index (QI) was designed as a gold standard to measure the quality of the medication process phases of the participating hospitals. To obtain this non-validated gold standard, five hospital pharmacists and five oncologists from different hospitals were requested to distribute 100 
Table 1 Description of the 10 paragraphs of the medication order entry and pharmacovigilance questionnaire

\begin{tabular}{|c|c|c|}
\hline Paragraph & Description & $\begin{array}{l}\text { No. of } \\
\text { questions }\end{array}$ \\
\hline 1. The hospital & Characteristics of hospital & 6 \\
\hline 2. Medication order entry system in general & Is there an electronic medication order entry system and who is entering the medication? & 3 \\
\hline 3. Pharmacovigilance in general & Dosage, interactions or contraindications & 6 \\
\hline 4. Laboratory values in general & Are laboratory values checked for medication surveillance in general? & 5 \\
\hline 5. Databases & What kind of databases are used in the hospital pharmacy? & 3 \\
\hline $\begin{array}{l}\text { 6. Medication order entry system for cytostatic } \\
\text { agents }\end{array}$ & $\begin{array}{l}\text { Is there an electronic medication order entry system for cytostatic agents and who is entering the } \\
\text { medication? }\end{array}$ & 6 \\
\hline 7. Pharmacovigilance for cytostatic agents & Is pharmacovigilance carried out for cytostatic agents? & 12 \\
\hline 8. Dose reduction in cytostatic agents & Is dose reduction visible and is it clearly stated that it concerns dose reductions? & 7 \\
\hline 9. Data transfer & Are data concerning cytostatic agents communicated? & 3 \\
\hline 10. Adverse events & Registration of AEs concerning cytostatic and non-cytostatic agents & 7 \\
\hline
\end{tabular}

points, according to their personal expert opinion, over 10 questions covering all medication phases.

By using the QI the real importance in clinical practice for the different questions could be assessed, meaning that the most important and relevant topic was assigned the most points while the least important topic was assigned the least points. There were no minimum or maximum points to assign per question, as long as the total points over all questions totalled 100 . The topics of these questions for cytostatic agents and non-cytostatic agents included the prescribing process, pharmacovigilance, acknowledgment of the medication processes to other healthcare professionals and registration of side effects.

In addition, an electronic questionnaire was developed which consisted of 58 multiple choice questions about the medication process. The electronic questionnaire was structured in 10 paragraphs and the most important points are listed in table 1 . This questionnaire was sent to five independent healthcare professionals ((hospital) pharmacists and physicians) to assess its completeness, clarity and appropriateness as an internal validation process. These five independent healthcare professionals were asked to review the questionnaire and their comments were used to optimise the questionnaire. No major adaptations had to be made.

The questionnaire was sent to hospital pharmacists in hospitals throughout The Netherlands. Eligible hospitals included in the study were Dutch hospitals administrating intravenous cytostatic agents. To compare the findings of the participating hospitals for the different topics of the questionnaire, a score system was developed by two healthcare professionals (hospital pharmacists). All of the questions were reviewed and scored by these two individuals independently. The questions for which no consensus was achieved were discussed by a third independent healthcare professional (hospital pharmacist).

Each question was scored from 0 to 2, where 2 represents the most optimal answer and 0 represents the least optimal answer. These scores were then weighted by using the QI to compare the scores in a standardised way. The mean values and 95\% CIs of the corrected scores were calculated using SPSS V.19. No additional statistical tests were performed.

Finally, to evaluate possible matched studies in the European setting, a PubMed search was performed.

\section{RESULTS}

\section{Quality index}

The five oncologists and five hospital pharmacists specified their key factors for the medication process. According to the given scores shown in table 2, there was consensus within the hospital pharmacists group and the oncologists group and between the groups. Some differences in opinion were found between hospital pharmacists and oncologists. The most striking differences concerned the use of laboratory values for pharmacovigilance and the communication to other healthcare professionals. Hospital pharmacists considered both topics to be more relevant than oncologists $(10.0 \%$ for hospital pharmacists vs $6.6 \%$ for oncologists concerning the laboratory values and $9.6 \%$ for

Table 2 Mean (SD) quality index score (\%) per topic

\begin{tabular}{|c|c|c|c|}
\hline Topic & Hospital pharmacist & Oncologist & Overall \\
\hline Pharmacovigilance general & $12.0(1.9)$ & $14.2(2.9)$ & $13.1(2.6)$ \\
\hline Laboratory values & $10.0(0.7)$ & $6.6(4.0)$ & $8.3(3.2)$ \\
\hline Use of one database & $10.1(1.4)$ & $10.9(3.3)$ & $10.5(2.4)$ \\
\hline Prescribing cytostatic agents-digital & $10.4(1.7)$ & $9.6(4.9)$ & $10.0(3.5)$ \\
\hline Prescribing cytostatic agents—standardised form & $10.1(1.9)$ & $10.6(8.3)$ & $10.4(5.7)$ \\
\hline Pharmacovigilance cytostatic agents & $10.8(0.8)$ & $13.6(2.6)$ & $12.2(2.4)$ \\
\hline Dose reduction visible for hospital pharmacist & $9.6(0.9)$ & $8.6(3.9)$ & $9.1(2.7)$ \\
\hline Communication to other healthcare professionals & $9.6(1.7)$ & $6.9(3.6)$ & $8.3(3.0)$ \\
\hline Registration of ADRs for cytostatic agents & $8.6(1.6)$ & $9.4(4.8)$ & $9.0(3.4)$ \\
\hline Registration of ADRs in general & $9.0(2.0)$ & $9.4(4.8)$ & $9.2(3.5)$ \\
\hline
\end{tabular}


Table 3 Hospital characteristics (mean number (SD))

\begin{tabular}{lllr}
\hline & Pharmacists & Beds & Patients* \\
\hline General hospitals $(\mathrm{n}=27)$ & $4.1(2.4)$ & $383(160.1)$ & $50(21.5)$ \\
Top clinical hospitals $(\mathrm{n}=23)$ & $7.0(1.6)$ & $708(204.3)$ & $122(75.4)$ \\
University hospitals $(\mathrm{n}=6)$ & $>10$ & $785(264.0)$ & $141(76.0)$ \\
Overall $(\mathrm{n}=56)$ & $5.9(2.8)$ & $575(272.5)$ & $85(64.9)$ \\
\hline
\end{tabular}

${ }^{*}$ Receiving cytostatic agents (per week).

hospital pharmacists vs $6.9 \%$ for oncologists concerning communication).

\section{Questionnaire}

The questionnaire was sent to hospital pharmacists in 80 hospitals; $56(70 \%)$ answered the questionnaire. Two hospitals answered the questionnaire twice and of the 22 that did not respond, 3 were not dispensing intravenous cytostatic agents.
The main characteristics of the hospitals that responded to the questionnaire are listed in table 3 and the main findings of the questionnaire topics are outlined in table 4.

Cytostatic agent prescription, predefined gifts and cycles, and pharmacovigilance in general are mainly performed manually, that is, using a written form, whereas these procedures are mainly done automatically for the non-cytostatic agents. Pharmacovigilance for interactions and contraindications is not routinely performed for cytostatic agents, while for noncytostatic agents this is a routine procedure.

If one looks at the use of laboratory values, there are some differences (figure 1). Forty-nine hospital pharmacists (88\%) indicated that all laboratory values such as kidney function, liver function and blood cell count are available, but they are not used when performing non-cytostatic medication surveillance.

\section{Correction by quality index: all hospitals}

The scores obtained from the questionnaire were weighted by the QI and then the results of the participating hospitals were

Table 4 Comparison between cytostatic and non-cytostatic agents

\begin{tabular}{|c|c|c|c|}
\hline & & Cytostatic agents (\%) & Non-cytostatic agents (\%) \\
\hline \multirow[t]{3}{*}{ Medication order entry system } & Electronic & 17 & 38 \\
\hline & Manual & 54 & 15 \\
\hline & Combination & 29 & 47 \\
\hline \multirow[t]{3}{*}{ Person who enters the medication in the medication order system } & Physician (Hospital) pharmacist & 71 & 52 \\
\hline & Nurse/pharmacy technician & 14 & 32 \\
\hline & Combination & 15 & 16 \\
\hline \multicolumn{4}{|l|}{ Method used to perform pharmacovigilance for: } \\
\hline \multirow[t]{4}{*}{ Interactions } & Automatic & 12 & 62 \\
\hline & Manual & 41 & 15 \\
\hline & Combination & 15 & 22 \\
\hline & No surveillance & 31 & \\
\hline \multirow[t]{4}{*}{ Contraindications } & Automatic & 7 & 34 \\
\hline & Manual & 55 & 33 \\
\hline & Combination & 10 & 24 \\
\hline & No surveillance & 28 & 9 \\
\hline \multirow[t]{4}{*}{ Overdosage } & Automatic & 23 & 47 \\
\hline & Manual & 53 & 10 \\
\hline & Combination & 24 & 59 \\
\hline & No surveillance & & 2 \\
\hline \multirow[t]{4}{*}{ Underdosage } & Automatic & 19 & 47 \\
\hline & Manual & 55 & 10 \\
\hline & Combination & 26 & 59 \\
\hline & No surveillance & & 2 \\
\hline \multirow[t]{4}{*}{ Total dose } & Automatic & 17 & 47 \\
\hline & Manual & 67 & 10 \\
\hline & Combination & 12 & 59 \\
\hline & No surveillance & 2 & 2 \\
\hline \multicolumn{4}{|l|}{ Person who performs the pharmacovigilance for: } \\
\hline \multirow[t]{4}{*}{ Interactions } & Hospital pharmacist & 29 & 48 \\
\hline & Physician & 26 & 3 \\
\hline & Combination & 16 & 45 \\
\hline & No answer & 29 & 4 \\
\hline \multirow[t]{3}{*}{ Contraindications } & Hospital pharmacist & 10 & 32 \\
\hline & Physician & 67 & 21 \\
\hline & Combination & 23 & 47 \\
\hline \multirow[t]{3}{*}{ Overdosage } & Hospital pharmacist & 33 & 37 \\
\hline & Physician & 3 & 3 \\
\hline & Combination & 64 & 60 \\
\hline \multirow[t]{4}{*}{ Underdosage } & Hospital pharmacist & 20 & 37 \\
\hline & Physician & 7 & 3 \\
\hline & Combination & 59 & 60 \\
\hline & No answer & 14 & \\
\hline \multirow[t]{3}{*}{ Total dose } & Hospital pharmacist & 33 & 37 \\
\hline & Physician & 27 & 3 \\
\hline & Combination & 40 & 60 \\
\hline
\end{tabular}




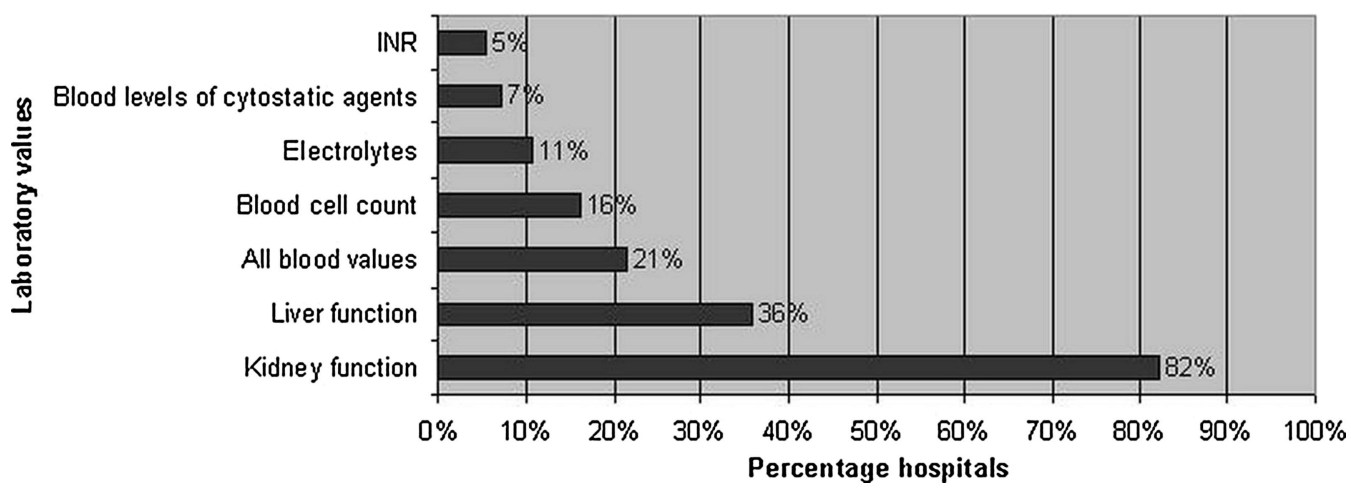

Figure 1 Use of laboratory values by hospital pharmacists. INR, international normalised ratio.

compared. The mean score of the hospitals was $47.2 \%$, with a minimum of $29.1 \%$ and a maximum of $72.5 \%$. The $95 \%$ CI of the mean score was 44.56 to $49.92 \%$. These results are reported in table 5 .

\section{Correction by quality index: score $>\mathbf{5 0} \%$}

Of the 56 participating hospitals, 22 (39.3\%) scored more than $50 \%$, considered to be the minimum score, taking into account the opinion of the healthcare professionals who performed the QI. In contrast to the results of all participating hospitals together, it seems that the general hospitals performed better when checking the $\geq 50 \%$ scores in comparison with top clinical and university hospitals.

\section{DISCUSSION}

Differences were found between hospital pharmacists and oncologists when evaluating the QI. We believe that the most plausible reason for these differences is that hospital pharmacists are often the ones who adapt doses according to the laboratory values, for cytostatics and non-cytostatics, and hence they gave a higher score to the specific question.

Oncologists are less likely to actively send medication information to other healthcare professionals, since this information is available in the electronic patient file. Other healthcare professionals working in the hospital can access this information when necessary. However, hospital pharmacists communicate

Table 5 General results

\begin{tabular}{llll}
\hline & & Score (\%) & $95 \%$ Cl (\%) \\
\hline Overall & General $(n=27)$ & 47.2 & 44.6 to 49.9 \\
Type of hospital & Top clinical $(n=23)$ & 46.2 & 44.0 to 52.3 \\
& University $(n=6)$ & 45.5 & 38.8 to 54.4 \\
Number of pharmacists & $<5(n=21)$ & 44.2 & 39.0 to 52.0 \\
& $5-9(n=26)$ & 49.6 & 40.1 to 48.3 \\
& $\geq 10(n=9)$ & 46.0 & 41.0 to 54.4 \\
Number of beds & $<400(n=18)$ & 49.0 & 43.6 to 54.5 \\
& $400-800(n=30)$ & 46.6 & 43.3 to 49.9 \\
Number of patients* & $>800(n=8)$ & 45.8 & 38.3 to 53.2 \\
& $<60(n=20)$ & 48.3 & 43.8 to 52.9 \\
& $60-120(n=15)$ & 48.8 & 43.0 to 54.6 \\
& $>120(n=10)$ & 44.8 & 39.2 to 50.3 \\
& NA $(n=11)$ & 45.5 & 39.6 to 51.3 \\
\hline${ }^{*}$ Receiving cytostatic agents (per week). & & \\
NA, not available. & &
\end{tabular}

frequently with first-line healthcare professionals (community pharmacists) and therefore consider this objective more important.

Most of the participating hospitals in this study use a combination of paper and electronic forms when prescribing noncytostatic agents and performing pharmacovigilance for these agents. For cytostatic agents, almost all hospitals prescribe, predefine cycles and gifts, and perform pharmacovigilance using paper forms. This also applies to dose reduction, as in most of the hospitals this is only visible in written form. In comparison, in one of the participating hospitals, all of these actions are performed electronically.

There were no major differences in questionnaire responses between general, top clinical and university hospitals. We consider this a positive point because it means that these hospitals are working in a comparable way, yet negative because this level is still insufficient.

Further investigation is needed to establish the relevance and incidence of interactions between non-cytostatic agents and cytostatic agents. These data may significantly change the current view of pharmacovigilance. In addition, prospective studies are necessary to realise the impact of these interactions and the consequences (necessity to take action: monitor, stop a specific medication and change doses/schemes).

In one of the hospitals, pharmacovigilance for intravenous cytostatic agents is an ongoing objective. It is performed by hospital pharmacists, physicians and nurse practitioners. The main characteristics of this specific objective are as follows: an electronic medication order entry system; electronically visible medication; electronic pharmacovigilance; availability of all blood values; complete digital patient database; electronic predefinition for cytostatic treatments and cycles; automatically calculated cytostatic dose; electronically performed cytostatic pharmacovigilance; digitally visible dose reduction; communication of intravenous cytostatic agent data to community pharmacies; and accessibility to home medication. ${ }^{14}$

There are differences when comparing the hospitals that obtained a score $\geq 50 \%$. In this group, a general hospital with 5-9 pharmacists, less than 400 beds and 60-120 patients receiving cytostatics seen weekly performed best, as outlined in table 5. A formal comparison of all the hospitals in The Netherlands is impossible since different systems for medication surveillance are used and no comparison can be made between the different systems.

To evaluate possible matched studies in the European setting, a PubMed search was performed. Nevertheless no comparable studies were found and no comparison could be made. 


\section{CONCLUSION}

Cytostatic agent prescription, predefinition for gifts and cycles, and pharmacovigilance are mainly performed using a written form with the risk that this could lead to human errors such as misapprehension, information loss and/or incomprehensibility.

Knowing the high toxicity and narrow therapeutic range of cytostatic agents, there is much work to be done to achieve the same pharmacovigilance level and quality that currently exists for non-cytostatic agents.

Introducing a fully digital system in hospitals may be the key for optimising pharmacovigilance, with the advantages of error reduction and/or more reliable traceability. Currently electronic vigilance systems are being implemented in Dutch hospitals, therefore the situation described here is expected to change in the near future.

\section{What this paper adds}

\section{What is already known on this subject}

- Pharmacovigilance for interactions and contraindications is currently not routinely performed for cytostatic agents, while for non-cytostatic agents this is a routine procedure.

\section{What this study adds}

- This study shows that there is still much work to be done to achieve the same pharmacovigilance level and quality that currently exists for non-cytostatic agents.

- This study describes the current situation in The Netherlands, referring to medication order entry systems and pharmacovigilance for cytostatic and non-cytostatic drugs.
Contributors All the authors contributed significantly to the publication. $\mathrm{BPCVO}$ and CMG contributed equally.

Competing interests None.

Provenance and peer review Not commissioned; externally peer reviewed.

\section{REFERENCES}

1 Riechelmann RP, Krzyzanowska MK, O'Carroll A, et al. Symptom and medication profiles among cancer patients attending a palliative care clinic. Support Care Cancer 2007;15:1407-12.

2 Riechelmann RP, Moreira F, Smaletz 0, et al. Potential for drug interactions in hospitalized cancer patients. Cancer Chemother Pharmacol 2005:56:286-90.

3 Scripture CD, Figg WD. Drug interactions in cancer therapy. Nat Rev Cancer 2006;6:546-58

4 Brewer T, Colditz GA. Postmarketing surveillance and adverse drug reactions: current perspectives and future needs. JAMA 1999:281:824-9.

5 Lindquist M. The need for definitions in pharmacovigilance. Drug Saf 2007;30:825-30

6 WHO. The importance of pharmacovigilance: safety monitoring of medicinal products. Geneva: World Health Organization, 2002. ISBN 9241590157.

7 Egberts TCG, Smulders M, de Koning FHP, et al. Can adverse drug reactions be detected earlier? A comparison of reports by patients and professionals. BMJ 1996:313:530-1.

8 Ray WA, Griffin MR, Avon J. Evaluating drugs after their approval for clinical use. N Engl J Med 1993;329:2029-32.

9 EMA. Data elements for transmission of individual case safety reports ICH E2B. London: EMA, 2005.

10 Lortie FM. Postmarketing surveillance of adverse drug reactions: problems and solutions. CMAJ 1986;135:27-32.

11 Venning GR. Identification of adverse reactions to new drugs. II (continued): how were 18 important adverse reactions discovered and with what delays? BMJ 1983:286:365-8.

12 Venning GR. Identification of adverse reactions to new drugs. I: What have been the important adverse reactions since thalidomide? BMJ 1983:286:199-202.

13 Venning GR. Identification of adverse reactions to new drugs. IV: Verification of suspected adverse reactions. BMJ 1983:286:544-7.

14 Stoop AP, Bal R, Berg M. OZIS and the politics of safety: using ICT to create a regionally accessible patient medication record. Int J Med Inf 2007;76:229-35.

Acknowledgements We would like to thank H A W van Onzenoort, H J Derijks, K Waizy, S J G M Ahlers, B G Kaufman, R J W van Kampen, N A J B Peters, G M J Bos, M $O$ den Boer, F A R M Warmerdam for establishing the gold standard, based on their personal expert opinion. 